Mots. Les langages du politique

102 | 2013

Les discours sur l'enseignement supérieur et la recherche

\title{
Des discours politiques au discours de l'évaluation. Autour de la réforme des formations universitaires
}

From policy discourses to discourses of assessment. On the reform of univer- sity curricula

De los discursos políticos a los discursos de la evaluación. Acerca de la reforma de la formaciones universitarias

\section{Sophia Stavrou}

\section{(2) OpenEdition}

Journals

Édition électronique

URL : http://journals.openedition.org/mots/21357

DOI : $10.4000 /$ mots. 21357

ISSN : 1960-6001

Éditeur

ENS Éditions

Édition imprimée

Date de publication : 2 septembre 2013

ISBN : 978-2-84788-414-2

ISSN : 0243-6450

Référence électronique

Sophia Stavrou, « Des discours politiques au discours de l'évaluation. Autour de la réforme des formations universitaires », Mots. Les langages du politique [En ligne], 102 | 2013, mis en ligne le 02 septembre 2015, consulté le 20 avril 2019. URL : http://journals.openedition.org/mots/21357 ; DOI $10.4000 /$ mots. 21357 


\section{Des discours politiques au discours de l'évaluation. Autour de la réforme des formations universitaires}

Parler de «discours officiel» de l'enseignement supérieur n'est pas une évidence tant la sacro-sainte liberté académique a toujours constitué un enjeu central. Pour autant, cette thèse paraît valable, surtout aujourd'hui, à l'aune du processus de mutation dans lequel s'inscrit l'Université depuis la fin du $20^{e}$ siècle. Cela passe par la production et diffusion d'un discours politique qui s'approprie le thème de l'enseignement supérieur et établit des orientations légitimes à travers une série de communications nationales et transnationales, de textes législatifs et réglementaires. Les politiques d'enseignement supérieur, qui connaissent un essor sans précédent, construisent en effet la nécessité du changement de l'Université vers une rationalisation socio-économique de son rôle. Ce discours officiel présente aussi un autre trait caractéristique : sa matérialisation et sa mise en œuvre par un système d'évaluation en cours d'institutionnalisation. C'est sur cet objet que se penche cet article.

L'avènement du phénomène de l'expertise dans tous les domaines de la vie sociale, et en particulier dans l'action publique, n'a pas laissé les sciences sociales indifférentes. Beaucoup de travaux scientifiques ont entrepris la tâche de décortiquer la relation complexe qu'entretiennent aujourd'hui politique et expertise. Dans le cas de l'enseignement supérieur, certaines recherches ont mis en évidence le déplacement des lieux de production des politiques, de la sphère politique vers des sphères technocratiques qui ancrent leur légitimation sur leur capacité d'expertise (Cussó, Gobin, 2008 ; Cussó, 2008 ; Garcia, 2009). Ces recherches montrent les transformations dans l'arène de production et leurs effets sur la nature et la forme du discours porté. Mais le phénomène expert acquiert aussi une deuxième dimension dans la réforme de l'Université. Cette dimension réfère aux lieux institutionnels de réappropriation du discours politique : les dispositifs d'évaluation des universités, introduits aux niveaux national et local. Dans cet article, nous nous attachons à démontrer le rôle que le discours de l'évaluation joue dans l'élaboration de la réforme de l'enseignement supérieur et comment son étude éclaire la question de la «circulation»

Université d'Aix-Marseille, Laboratoire méditerranéen de sociologie, MMSH stavrou_sofia@yahoo.fr 
(Gobin, Deroubaix, 2010) lexicale et sémantique des normes. Nous illustrons cela à travers l'exemple de la mise en œuvre de la politique de refonte des formations universitaires en France.

Le discours de l'évaluation ou expert, qui ne se prête pas à l'observation en tant que tel, est ici reconstruit à partir de divers éléments : les procédures et les outils, les activités des acteurs qui le mettent en œuvre et les relations qui s'établissent entre eux. Leur examen a été rendu possible par une analyse approfondie d'un corpus de données textuelles recueillies entre 2007 et 2011. Il s'agit de vingt-et-un récits de pratique d'agents institutionnels ainsi que de documents officiels mobilisés et/ou produits au cours de l'évaluation ${ }^{1}$. Nous faisons l'hypothèse, selon les principes défendus par Jean Molino (1997), que ces données discursives deviennent la «trace de la matérialité » formelle et sociale du phénomène observé. Elles constituent la matérialité des pratiques et de la réflexivité des acteurs sociaux et de l'institution sur ses activités et sur les relations entre ses membres.

Dans le contexte actuel, les études des discours de l'enseignement supérieur, auxquelles nous apportons notre contribution, présentent un intérêt crucial. Si les recherches sur le processus de réforme de l'enseignement supérieur ne peuvent pas faire l'impasse sur un tel objet, c'est parce que l'émergence de ces «discours» fait partie intégrante du phénomène observé. D’une part, c'est la prolifération de textes officiels qui initie et cadre ce processus. D'autre part, l'usage du discours et la place de l'échange verbal, notamment écrit, s'accroissent. On assiste à une professionnalisation de l'activité de production de discours - supports des échanges inter et intra-institutionnels - qui se développe avec les nouveaux modes de gouvernance des universités. Si l'on accepte que les politiques sont des régimes pragmatiques spécifiques de vérité et de valeur, on peut argumenter avec Stephen Ball (2008) que les discours à travers lesquels les politiques parlent et les discours qui parlent de ces politiques participent à la création des conditions de leur acceptation et de leur mise en œuvre.

1. Ce corpus de données a été constitué et analysé dans le cadre d'une recherche doctorale sur l'objet des transformations curriculaires dans les universités françaises (Stavrou, 2012). Le corpus restreint sur lequel s'appuient les résultats développés dans cet article est composé de huit entretiens auprès d'agents nationaux, experts et délégués scientifiques de l'Agence d'évaluation de la recherche et de l'enseignement supérieur, ainsi que de treize entretiens menés entre janvier 2007 et mai 2011 auprès d'agents universitaires locaux, membres des équipes de direction - vice-présidents, chargés de mission - et membres du Conseil des études et de la vie universitaire de six universités réparties sur le territoire français - Sud-Est, Est, Ouest, Paris Centre et appartenant aux quatre vagues de contractualisation - A, B, C, D. Il comprend également une série de textes officiels : textes législatifs, circulaires d'orientation de la Direction générale de l'enseignement supérieur, de la «littérature grise » interne, grilles d'expertise et un échantillon de rapports d'évaluation des formations. Ces données ont été passées au prisme d'une première analyse thématique et d'une analyse centrée sur le discours, à l'aide des outils de la théorie de l'énonciation et de la pragmatique linguistique. 


\section{Rationalisation des missions et rationalisation des activités de l'Université}

Nous souhaitons, au préalable, présenter les transformations qui s'opèrent dans le système d'enseignement supérieur. Ce changement se développe sur deux plans, le premier dans la définition du rôle de l'institution universitaire, le second dans l'organisation de son fonctionnement, mais les deux sont interdépendants.

\section{Discours politique et institutionnalisation d'un modèle de projection}

À partir de la fin de la deuxième moitié du $20^{e}$ siècle, se construit et se stabilise progressivement dans le discours politique une fonction instrumentale de l'enseignement supérieur, directement liée à l'économie. Pour le sociologue de l'éducation Basil Bernstein (2007), si plusieurs modèles pédagogiques continuent à s'affronter au sein du champ éducatif, la transformation fondamentale dans le domaine des politiques éducatives réside dans l'institutionnalisation d'un modèle qui valorise la production d' «identités de marché». Cela renvoie à la nouvelle valence que prend l'objectif de l'intégration des individus dans le marché, générant un principe officiel de «projection $»^{2}$ des formations universitaires et de leurs savoirs sur les marchés socio-économiques, en tant que principe qui oriente les pratiques dans la construction curriculaire.

L'analyse des textes officiels montre que, du point de vue de son contenu, le discours politique sur l'enseignement supérieur se caractérise par un relatif consensus polyphonique autour de la sémantique des orientations officielles. Ce discours opère une classification de principes - le changement par opposition au maintien - en banalisant le thème du changement au-delà de tout débat politique conflictuel. Il met en relation des catégories telles que connaissance et économie, en définissant le sens précis de cette relation par la mobilisation d'un principe de projection. D'une part, il transforme la politique de l'enseignement supérieur en une politique publique-substitut, c'est-à-dire mise au service d'autres politiques publiques - de l'emploi et du développement économique. D'autre part, il établit des orientations officielles de l'action en vue d'une professionnalisation des diplômes.

Ce discours est produit à l'intérieur du contexte français par l’État, par le biais

2. Le concept de «projection» désigne une centration sur la valeur externe des formations universitaires et de la connaissance qu'elles permettent d'acquérir, différent de l'« introjection » qui caractérise une logique centrée sur le développement des disciplines académiques ou sur la fonction de contrôle symbolique pour la construction d'une culture commune chez les individus (Bernstein, 2007). Dans notre étude (Stavrou, 2012), il réfère à un principe qui se définit dans le discours officiel, lequel oriente les opérations de constitution des objets de formation, de sélection et d'organisation des savoirs. 
du changement des missions officiellement assignées à l'enseignement supérieur. Amorcé dans la loi Faure de 1968, ce changement se concrétise avec la loi LRU en 2007 qui marque un tournant crucial : elle institutionnalise notamment la mission de «l'orientation et l'insertion professionnelle» et contribue à redéfinir par là l'intérêt des formations supérieures. Cette politique se développe progressivement. Entre 1980 et aujourd'hui, une multitude de rapports d'experts - chercheurs mais aussi représentants du monde socio-économique - participent à l'élaboration du sens des orientations officielles. Les recommandations tendent vers une extension de la fonction de professionnalisation des diplômes, pardelà les formations dites professionnelles, aux diplômes universitaires généraux et aux filières académiques classiques et posent la nécessité de repenser les objets et les contenus d'enseignement. Pour sa part, la Direction générale de l'enseignement supérieur y joue aussi un rôle décisif, par la transformation du mode de pilotage de l'offre de formation : les maquettes nationales par discipline sont abandonnées, au profit d'une régulation par objectifs définis en amont. Cette politique incitative contribue ainsi à thématiser la professionnalisation des diplômes dans les circulaires d'orientation qui encadrent les pratiques.

La définition des orientations s'inscrit aussi dans un espace européen et international de conception des cadres sociocognitifs de l'action publique (Charlier, Croché, 2003; Croché, 2006; Ravinet, 2009). Cet espace, pluriel, se complexifie dans le temps pour inclure divers groupes d'acteurs - ministres des gouvernements nationaux, associations syndicales, organisations patronales, agences d'expertise, instances politiques européennes, organismes internationaux. L'inscription du projet européen de Bologne dans la stratégie du développement économique et social, posée par le Conseil européen à Lisbonne, fait glisser le projet vers le vocable d'une "économie de la connaissance». L'éducation et la formation deviennent un enjeu d'investissement dans les ressources humaines et le système d'enseignement supérieur doit se transformer de manière à améliorer les conditions d'« employabilité» des individus et d'une «croissance économique durable» (Conférence des ministres, 2003). Dans les communiqués du processus de Bologne sont définis des objectifs stratégiques pour l'offre de formation : la «diversification» des programmes en fonction des «profils» des publics et de la diversité des «besoins» du marché du travail, le renforcement de la mission d' «insertion et d'orientation professionnelles» des individus, et le développement du «partenariat et de la coopération avec les employeurs ». Les positions de la Commission européenne, dont le ton est déjà donné dans ses deux Livres blancs publiés dans les années quatre-vingt-dix, soulignent aussi le rôle des systèmes d'éducation et de formation dans la stimulation de la croissance économique et vont dans le sens d'une impulsion politique de la «modernisation » des universités, articulée à ce rôle3 (SEC [2011] 1063).

3. Roser Cussó (2008) montre, à travers une analyse lexicométrique des communications de 
Notre ambition ici est moins d'établir un bilan des acteurs qui participent à la réforme de l'enseignement supérieur', que de mettre l'accent sur les spécificités de la nature de ce discours politique dont la production se réalise dans l'interaction entre ces acteurs. Si l'enseignement universitaire a toujours eu des visées plus ou moins professionnalisantes (Lessard, Bourdoncle, 2002), ce discours politique génère aujourd'hui des nouveautés :

- sa thématisation : la fonction professionnalisante de l'enseignement universitaire devient un objet du discours politique. Elle est soumise à un processus de substantialisation linguistique et cognitive et de transformation en enjeu politique;

- sa publicisation étendue : ce thème fait l'objet d'une masse considérable de documents. Il devient même la mission propre de certaines agences nationales ou européennes (Commission de la professionnalisation des enseignements supérieurs du Haut Comité éducation-économie, Commission du débat national université-emploi, Forum université-entreprise créé sous l'initiative de la Commission européenne).

Quoiqu'il établisse des normes d'ordre politique, ce discours se présente dans sa forme comme dépourvu de caractère contraignant; il est discours de recommandation, incitant à une amélioration rationnelle de l'état des choses. En revanche, dans sa dimension illocutoire (Austin, 1970), la production de ce discours politique apparaît comme un acte social d'appropriation du contrôle sur l'enseignement supérieur et sur son changement. II s'approprie la capacité et la légitimité de définir l'intérêt du système éducatif, ainsi que les solutions pratiques pour le réaliser. Ainsi, l'acte même de sa production et de sa diffusion, non seulement stabilise le contenu sémantique des principes, mais régule aussi la configuration de l'espace de réflexion et d'action sur l'Université. Il donne à l'institution universitaire le statut de «destinataire» des orientations véhiculées.

\section{Instauration d'un champ expert et pilotage rationalisé}

Si la classification sémantique des définitions du changement pour l'Université se réalise par et dans le discours politique, le cadrage pratique de l'action

\footnotetext{
la Commission de 1995 à 2003, la grande fréquence des occurrences des mots renvoyant au «changement» des systèmes d'enseignement supérieur et le lien systématiquement établi avec l'«adaptation » des systèmes au marché de l'emploi.

4. Les contributions de ces acteurs diffèrent aussi bien à l'intérieur qu'entre les deux scènes, nationale et transnationale. Les travaux de Sarah Croché $(2006,2009)$ montrent par exemple la distinction des rôles à l'intérieur de l'espace européen, entre le Conseil de l'Europe, la Commission européenne et les ministres de l'Enseignement supérieur des pays engagés dans le processus de Bologne. De même, d'autres acteurs internationaux qui participent à la construction de la politique de l'enseignement supérieur devraient être mentionnés ici, tels que l'OCDE, l'Unesco, la Banque mondiale ou l'OMC.
} 
se fait à partir d'un système d'évaluation de la «qualité », récemment mis en place et instauré par le discours politique lui-même.

L'expertise émerge, en France, dans le cadre de l'instauration d'un nouveau mode de pilotage: une politique contractuelle, régulant les relations entre l'État et les universités sur une base davantage stratégique et prévisionnelle (Musselin, 2001). Initiée en 1984, la politique contractuelle s’inscrit dans les textes législatifs en 2007 , sous le vocable de «nouvelle gouvernance » des universités (Loi n²007-1199). C'est dans ce contexte que naît le « nouveau dispositif d'évaluation » (DGES, 2005), en interaction forte avec les textes européens. Celui-ci annonce le passage d'une politique réglementaire à une politique davantage évaluative, laquelle associe, paradoxalement, «autonomie» - des établissements - et accroissement du contrôle par l'État. La réforme des curricula se concrétise ainsi dans le processus de contractualisation de l'offre de formation des universités.

À l'intérieur de chaque établissement c'est l'ensemble des instances universitaires qui est mobilisé pour l'examen des maquettes des formations, allant des directeurs d'UFR aux chargés de mission, aux vice-présidents délégués et à l'équipe de direction de l'université. La responsabilité spécifique incombe, néanmoins, aux Conseils des études et de la vie universitaire (CEVU) et à leurs organes annexes. À son origine, le CEVU répond essentiellement à une ambition démocratique, liée à la participation de tous les représentants de la population universitaire à l'organisation des études et à la liberté locale de la planification pédagogique, héritage de Mai 1968. En revanche, avec la mise en place de la politique contractuelle, du schéma LMD (2002) et plus tard de la loi LRU (2007), cette structure s'est progressivement constituée en champ d'expertise. Elle a acquis des pratiques d'expertise des projets de formation, intervenant en amont de l'évaluation des formations par les instances nationales et de leur habilitation par le ministère.

Au niveau national, l'évaluation est réalisée parl'Agence d'évaluation de la recherche et de l'enseignement supérieur (AERES), mise en place en 2006. Celleci succède aux deux conseils nationaux d'évaluation établis par la loi Savary de 1984, ainsi qu'à la Mission scientifique, technique et pédagogique du ministère, laquelle assure jusqu'en 2006 l'examen des projets de formation. Instituée en " autorité administrative indépendante », elle comprend des experts désignés ou autodésignés, enseignants-chercheurs, chargés de la rédaction de rapports d'évaluation sur chaque formation. Par rapport aux anciennes agences, la création de l'AERES marque les débuts d'une «professionnalisation » de l'activité d'évaluation. Car en son sein se développent et se formalisent des outils et des critères d'évaluation qui sont diffusés aux universités.

Ce champ expert ne renvoie pas à un champ homogène, car les modalités de l'activité d'expertise et ses normativités varient selon les établissements et les groupes considérés. Mais il réunit un groupe d'agents avec des activi- 
tés spécifiques et dont la présence marque l'installation officielle d'un champ extérieur au champ pédagogique, ayant droit d'intervention dans les curricula. À la sortie du $20^{e}$ siècle, on voit ainsi se cristalliser une nouvelle configuration triadique comprenant le pédagogique, le politique et l'évaluation.

\section{Le discours de l’évaluation comme médium pratique}

Nous avons tenté d'analyser ce discours de l'évaluation afin de saisir comment l'expertise, en tant que «dispositif technique et social» qui permet d'opérationnaliser l'action publique (Lascoumes, Le Galès, 2004), contribue au changement proclamé des curricula universitaires et, en particulier, à la mise en place du modèle de projection.

\section{Une continuité dans la définition des objectifs}

Il existe des orientations, reconnues comme légitimes, qui constituent le cadre normatif de l'action sur les curricula. Ce sont celles qui apparaissent dans les documents de cadrage pour la fabrication et l'expertise des maquettes, et qui sont évoquées lors des entretiens en tant que catégories officielles d'évaluation des programmes. Parmi les différents objectifs, celui de la professionnalisation et, plus largement, du rapprochement Université-marché constitue un principe essentiel. Ce dernier devient opérant en tant que critère d'évaluation. Il stabilise à la fois la nécessité de cette mise en relation et son sens spécifique. Les indicateurs mobilisés présupposent une mise en relation entre le curriculum et le marché socio-économique, objet sur lequel portent les évaluations : les partenariats avec les tissus socio-économiques, la participation d'intervenants professionnels, la mise en place d'une politique de stages, etc. Ils sousentendent aussi une relation de correspondance entre le curriculum et des domaines d'activité professionnelle, en évaluant le degré de projection de la formation vers des emplois, à travers les débouchés et les compétences visées.

Une forte continuité se dessine entre le discours de l'AERES, celui des instances universitaires et le discours politique. On peut constater une stabilité intertextuelle quant aux normes de qualité, ainsi qu'une forte homogénéité du lexique employé pour décrire ces normes. Au-delà de leur sémantique, ce sont aussi les formes signifiantes de ces injonctions, les mêmes «formules» (KriegPlanque, 2009) qui se stabilisent et qui circulent entre espaces institutionnels5. Le discours des experts interviewés est fortement marqué par l'expression d'un «dialogisme» (Bakhtine, 1977). Ceux-ci font apparaître fréquemment dans leur

5. L'exemple de la formule «adossement aux milieux socio-économiques » apparaissant dans les circulaires de la DGES et que nous retrouvons aussi dans les Notes de cadrage, la Fiche d'expertise et les rapports d'expertise produits par l'AERES illustre bien cette circulation. 
récit un discours second, dont ils ne sont pas énonciateurs. Sans que cela préjuge de leur adhésion, l'emploi de ce discours rapporté souligne pour les locuteurs l'existence d'un discours par rapport auquel ils sont amenés à se positionner dans leur pratique.

Le ministère dit il faut que vous remplissiez la fiche RNCP. Donc il faut aller sur le web et voir ce que c'est une fiche RNCP. Et donc aller sur les sites des compétences professionnelles, voir, chercher les codes. (Expert, membre élu CEVU) ${ }^{6}$

Alors mon travail ça a été d'abord de bien m'imprégner des rapports qui ont été faits, notamment par Jolion sur le master et Dizambourg sur la licence. Donc m'imprégner de leurs recommandations pour voir quelle traduction je peux en faire localement. L'intention c'était de mettre en évidence de manière très concrète dans les nouveaux cursus, dans le nouveau quadriennal, des éléments de professionnalisation des étudiants. (Rapporteur désigné, Commission des formations, organe annexe au (EVU)7

Mais la question de la circulation des normes ne se résume pas au repérage d'un contenu identique dans les deux discours, politique et expert. Ce qui paraît plus intéressant à saisir, c'est la manière dont s'établissent les conditions de cette circulation et ses enjeux, et qui sont à rechercher dans les modalités de mise en œuvre de l'acte évaluatif. Nous évoquerons dans la suite de l'article deux traits fondamentaux et interconnectés: d'une part, la formalisation des normes de la qualité dans des documents écrits, d'autre part, la prédominance de la fonction régulatrice dans l'acte évaluatif.

\section{La formalisation normative par les documents}

Comprendre ce qu'est l'expertise en tant qu'activité est indissociable de la question de la nature des documents experts et de leur usage. Ces documents, que nous qualifions d'outils de l'expertise, présentent un certain nombre de catégories et d'indicateurs à partir desquels l'évaluation d'une formation doit être réalisée. En ce sens, ils formalisent par l'écrit les normes de qualité pour les programmes.

À partir de la création de l'AERES, ces documents sont mis au centre du processus d'expertise. Pour les experts nationaux, quand il s'agit, au cours des entretiens, de décrire leur activité, il y a une référence constante à la «Fiche d'expertise», laquelle acquiert une fonction officielle de loupe d'observation des dossiers. Dans les extraits ci-dessous, l'emploi des verbes remplir, appliquer, les modalisateurs falloir et devoir dévoilent le rapport des experts à ces documents, ainsi que la division du travail entre leur usage et leur production.

6. Entretien réalisé en novembre 2008, Université de la vague de contractualisation B. Je souligne, dans cette citation et dans celles qui suivent.

7. Entretien réalisé en mai 2010, Université de la vague de contractualisation A. 
L'objectif est de remplir pour ces spécialités voire ces parcours le document que vous avez là, c'est-à-dire la fiche d'expertise d'une mention. Alors, par rapport à ce tableau-là il faut mettre chaque fois une note sous la forme d'une lettre, qui peut aller de $C$ à $A$. Pour chaque case, on doit insérer des commentaires. Et donc on travaille sur la base de fichiers informatiques qui nous ont été envoyés par l'AERES. $\left(\right.$ Expert AERES) ${ }^{8}$

Pour l'expertise réalisée au sein des universités, les documents écrits remplissent une double fonction. Ils constituent les outils sur lesquels les experts s'appuient pour mener leur évaluation. Mais ils s'adressent aussi aux composantes pédagogiques et orientent par là la fabrication des maquettes.

La création de l'AERES marque en effet un tournant dans l'usage de ces documents. Tandis que dans la première période post-LMD les universités voient dans l'abandon de maquettes disciplinaires nationales la possibilité d'inventer les leurs, la deuxième période, post-AERES, est caractérisée par une adoption progressive des «fiches d'expertise » publiées par l'agence nationale. Dès lors que les critères d'évaluation nationaux sont rendus publics, la question de leur utilisation par les instances locales s'impose. Soit l'activité locale de production est complètement abandonnée, soit cette activité a lieu, mais dans ce cas elle est projetée sur ces référents préexistants. On passe d'un cadrage sous forme politique à un cadrage sous forme technico-pratique, du moins dans l'appréhension qu'ont les agents universitaires des cadres institutionnels qui structurent leurs activités. Ce changement peut être révélé par la confrontation de deux discours, portés par les vice-présidents du CEVU d'une même université mais ayant exercé leur mandat à deux moments différents :

C'est le ministère qui expertise et ensuite il donne l'habilitation. Donc, avec un senti et puis avec des discussions qui ont été faites avec le ministère on sentait bien la philosophie générale... et donc on a essayé que ça rentre dans la philosophie générale et que ça ne soit pas n'importe quoi. Donc, nous avons expertisé, nous, des dossiers qui étaient présentés par des collègues ou par des départements. Nous avons demandé des changements, nous avons vérifié qu'il y avait un respect de règles que nous avions émises. (Vice-président CEVU 2004-2007)9

Alors on va faire un guide de l'expert, on ne va rien inventer, hein? C'est-à-dire qu'il va se fonder aussi un peu sur ce que regardent les experts de l'AERES quand ils évaluent... des choses très techniques, hein? [...] Le ministère ne donne pas de cadrage de ce point de vue-là. En revanche, celle qui donne un cadrage c'est l'AERES. Elle envoie... enfin, sur son site sont téléchargeables d'une part des documents précisant le contenu que doivent avoir les dossiers [...] Et aussi elle publie la fiche d'autoévaluation, enfin la fiche d'évaluation qu'elle va donner à ses experts pour évaluer le dossier, en nous disant si vous voulez, vous, pour votre bilan vous fonder sur ces différentes questions que les experts vont poser à votre dossier, ça peut être pour

8. Entretien réalisé en juin 2010.

9. Entretien réalisé en janvier 2007, Université de la vague de contractualisation B. 
vous une manière de rédiger le dossier. Donc, ils nous donnent des indications en fait. Chaque fois il faut chercher les documents. (Vice-président CEVU 2011-2014) ${ }^{10}$

Le premier enquêté appréhende le discours officiel comme une philosophie générale, un projet politique qui s'impose de manière indirecte. L'utilisation du verbe sentir révèle le caractère non clairement défini de ce cadrage, mais aussi sa non-stabilité. Si l'on se rapporte maintenant au second entretien, la souveraineté du ministère s'efface derrière la figure de l'AERES, mise en scène comme acteur principal de la procédure de contractualisation. Le contrôle est moins exprimé dans un schéma opposant le politique-national au pédagogique-local que par la figure d'un dispositif sociotechnique matériel.

La formalisation des normes par l'écrit remplit en effet trois fonctions principales. La première c'est qu'elle participe à la stabilisation d'une hiérarchie des préférences. Elle fige une classification spécifique des normes de production et d'évaluation des curricula, en particulier sur l'obligation de leurs objectifs et de leurs résultats, tant potentiels que mesurés. En inscrivant ces catégories d'appréhension dans les documents, la formalisation les rend nécessaires, puisqu'elle oblige à se confronter à celles-ci et à y répondre.

Sa deuxième fonction c'est qu'elle transforme des normes reposant sur des choix politiques en normes techniques et procédurales, qui définissent comment mettre en œuvre la production des programmes de manière efficace et garantir un bon ajustement aux cadres officiels. Les documents sont des outils techniques, dans le sens où ils consistent en la transmission d'un ensemble de savoir-faire pour la réalisation d'une activité pratique. Mais du moment où ils se stabilisent, ils deviennent aussi «objets de connaissance» (Akrich, 1987).

Corrélative des deux autres, la troisième fonction de ce système formel c'est qu'il rend possible la circulation de ces normes au sein des institutions et entre elles. Il permet la production d'une stabilité discursive dans les textes fabriqués aux divers niveaux institutionnels. Et, par là, il s'ouvre à la production d'une conscience théorique commune ${ }^{11}$ sur la définition des problèmes de l'enseignement universitaire, tels que la professionnalisation des diplômes. Comme le soulignent Pierre Lascoumes et Patrick Le Galès (2004), les instruments stabilisent une problématisation et une représentation spécifique de l'enjeu qu'ils traitent, en même temps qu'ils produisent des effets d'inertie, c'est-à-dire de résistance à leur dé-stabilisation. Dans ce sens, la fabrication d'instruments, par la formalisation d'une grille d'évaluation, joue un rôle clé

10. Entretien réalisé en décembre 2009, Université de la vague de contractualisation B.

11. La mobilisation du concept de commentaire chez Michel Foucault permet de rendre compte de ce procès de production des discours institutionnels, qui opère des décalages entre texte premier et texte second, susceptibles de construire des discours nouveaux, mais qui n'a pour rôle enfin que de dire ce qui était déjà là. Le commentaire conjure le hasard du discours : "Il permet bien de dire autre chose que le texte même, mais à condition que ce soit ce texte même qui soit dit et en quelque sorte accompli.» (Foucault, 1971, p. 27) 
dans la mise en place de la politique de l'enseignement supérieur à l'intérieur du contexte national français ${ }^{12}$.

Selon la conceptualisation de Jean de Munck (1999), la limite principale des systèmes formels c'est qu'ils ont la capacité de mettre sous silence la division sociale du travail qui les a générés, aussi bien que le contexte sociohistorique qui les valorise. En revanche, ils présentent aussi un triple aspect de «productivité », car ils peuvent donner lieu à l' "élucidation » d'un domaine de sens, à sa "généralisation», enfin à sa "transmissibilité», en explicitant et diffusant les règles. Ce sont ces propriétés qui peuvent potentiellement permettre aux catégories mobilisées pour le changement de l'Université de devenir objet de débats, de réflexions collectives. L'enjeu réside dans l'usage qui est fait de ces documents experts dans l'activité d'évaluation. C'est cet usage que nous tentons de questionner dans la section suivante.

\section{Un acte régulateur: l'activité évaluative guidée parle principe d'efficience}

La manière dont l'acte évaluatif est mis en œuvre joue aussi dans la production d'une continuité entre le discours politique et le discours expert. L'expertise apparaît comme une activité ayant une conséquence politique, au sens où elle produit un classement social des formations universitaires. Experts des CEVU et experts de l'AERES émettent des avis et attribuent des notes qui jouent le rôle de signal de la qualité d'une formation, justifiant ou sanctionnant son existence, en amont de la décision d'habilitation. Mais l'expertise acquiert aussi une conséquence pratique, car elle contribue à instituer des manières de faire. Elle opère matériellement sur la production des textes pédagogiques. Elle participe à une classification d'objets et de significations, à travers les appréciations/dépréciations que réalise l'expert, faisant la part entre «bonnes » et «mauvaises» pratiques.

Le champ expert universitaire, se situant au plus près des producteurs, mène une action plus directe sur les maquettes. L'opération d'autorisation/ rejet des projets par le CEVU n'apparaît que comme ultime solution, tandis que le propre de l'activité pratique de cette agence semble résider dans un travail de correction des maquettes, dans une logique de réussite de l'établissement. Dès lors, la figure de l'expert n'est plus seulement celle de « juge » de la qualité, mais aussi celle d'un agent qui agit sur les curricula :

12. Ravinet (2011) démontre la force des instruments telle qu'elle s'exprime également à l'échelle européenne, au niveau de la coordination du processus de Bologne. Le développement de la pratique du stocktaking - la fabrication d'une grille de collecte d'informations pour l'ensemble des pays - est révélatrice de la manière dont ce type d'instrument permet de matérialiser et d'orienter la dynamique collective, ainsi que de recomposer les mécanismes de la contrainte et de l'engagement dans un processus dépourvu de sanction. 
Je veux bien qu'on fasse le ménage parmi nos formations, nous et pas le ministère, qu'on ne renvoie pas tout! Mais, dans un premier temps moi je ne peux pas conseiller aux experts de mettre C d'emblée. Il faut vraiment essayer de sauver ce qui est sauvable! (Vice-président CEVU) ${ }^{13}$

Au bout du compte on vote, donc on prend une décision. Simplement, si le travail est bien fait et je crois qu'il a été fait là, quand on est arrivé au stade du vote il n’y avait plus de problème. C'est-à-dire que vraiment tout ce qui pouvait empêcher un vote positif avait été normalement réglé avant. (Expert, membre élu CEVU) ${ }^{14}$

Les experts appréhendent leur activité à travers le couple notionnel «travail-utilité ». C'est-à-dire comme activité pratique et comme activité efficiente qui a la propriété de "régler les problèmes». Ce travail sur les maquettes signale une action qui se réalise sur un projet qui le fait passer d'un état $A$ à un état $B$, par une action seconde de correction qui s'ajoute à celle de la production des programmes. Le travail d'expertise repose en effet sur une conception spécifique de l'amélioration, régulatrice. Non seulement l'état de qualité visée est prédéterminé, mais, de plus, seuls les projets considérés comme «défectueux» peuvent avoir accès au procès d'amélioration.

Il y a un master sur lequel j'ai rapporté, où le contenu est très bien, très intéressant et puis quand ils renseignent la rubrique sur quels sont les débouchés ils disent simplement que nos étudiants pourront travailler dans des écoles de langues. Ça fait très... c'est très décevant. Donc, moi, l'avis que j'ai rendu c'est qu'il faut améliorer ça bien sûr. C'est le sentiment d'une maquette qui ne sait pas bien se vendre quoi. Voilà, c'est dommage... ça ne correspond pas à ce que demande le ministère. (Membre élu CEVU) ${ }^{15}$

Ce principe de correction structure les relations interindividuelles entre experts et porteurs de projet, qui tendent vers un rapport de pédagogisation (Bernstein, 2007), centré sur l'aide et l'explicitation. Les uns - les agents pédagogiques - sont considérés comme des agents qui ne possèdent pas le savoirfaire nécessaire pour la production d'une «bonne maquette»; les autres - les experts -, en tant que détenteurs du savoir-faire légitime, tentent de modifier les pratiques des premiers. Les instances universitaires s'inscrivent ainsi dans une logique portée sur la valeur d'échange de ce travail d'évaluation, c'est-àdire l'habilitation et le financement par l'État.

Les experts de l'AERES, eux, mènent leur activité d'expertise de manière déconnectée des composantes pédagogiques. Dans ce cas aussi, on constate que la mise en œuvre de cette activité est davantage orientée par son versant régulateur, à savoir l'aide à la décision politique en vue de l'habilitation d'une formation. Elle repose sur une certaine conformité de l'expert au discours insti-

13. Entretien réalisé en décembre 2009, Université de la vague de contractualisation B.

14. Entretien réalisé en avril 2010, Université de la vague de contractualisation D.

15. Entretien réalisé en février 2010, Université de la vague de contractualisation B. 
tutionnel déclaré, à l'égard des objets à évaluer et des indicateurs, et elle attribue ainsi une place privilégiée aux outils et savoirs experts.

L'adaptation de l'évaluateur aux catégories officielles expertes apparaît notamment dans le contenu des rapports produits. L'analyse automatique des rapports d'expertise des formations ${ }^{16}$, à l'aide du logiciel Alceste, a permis de dégager quatre champs lexicaux qui constituent les points d'ancrage du discours évaluatif, selon les cooccurrences des mots qui figurent dans le corpus. On constate, en effet, que ces champs semblent caractérisés par un degré élevé d'adéquation aux rubriques d'évaluation prescrites par l'AERES : ils correspondent à quatre domaines thématiques, celui du dossier expertisé, celui de l'enseignement, celui du marché du travail, et celui de l'ancrage géopolitique et institutionnel des formations ${ }^{17}$. L'examen du type d'activité discursive réalisée par les experts dans ces rapports permet d'approfondir ce constat. On peut observer la mobilisation de trois registres discursifs ${ }^{18}$ : un registre informatif, qui caractérise les énoncés dans lesquels l'évaluateur se contente de décrire la formation à partir d'informations contenues dans les maquettes; un registre axiologique, où l'évaluateur qualifie comme point positif ou négatif un élément de la formation; un registre prescriptif, où l'évaluateur suggère directement à la composante pédagogique des modifications. C'est le deuxième registre qui est le plus fréquemment mobilisé, c'est-à-dire des énoncés au sein desquels l'expert pose comme objet de référence les objets légitimes à évaluer et accomplit un acte d'axiologisation des manifestations de ces objets. Pour reprendre les concepts de Ducrot (1984), chaque fois que l'expert procède à un acte discursif d'axiologisation, il met en scène à la fois un niveau "présupposé», des contenus présentés comme allant de soi, déjà validés et soustraits à toute contestation, qui relèvent de l'officiel, et un niveau "posé », des contenus explicites et soumis aux éventuelles objections de l'allocutaire, qui relèvent de l'interprétation de l'expert. Dans l'énoncé ci-dessous, le présupposé renvoie au fait que la relation formation-emploi peut être établie et qu'elle constitue une norme légitime pour la construction des formations et donc de leur évaluation.

16. Il s'agit de l'ensemble de 253 rapports d'expertise des mentions de master du domaine SHS, disponibles sur le site de l'AERES en décembre 2009 (Vagues de contractualisation B, C, D).

17. La Classe 1 concerne la présentation du dossier de la formation, les informations qu'il donne ou qu'il ne donne pas (mots les plus représentatifs : informations, présentation, précisions, équipe pédagogique, manque). La Classe 2 englobe une série de mots qui concernent l'organisation des enseignements, à savoir le curriculum interne (UE, semestre, option, volume horaire, mutualisation, stage). La Classe 3 concerne directement la mise en relation entre la formation et l'emploi ciblé ou le marché du travail plus largement (métier, gestion, collectivités territoriales, conception, public, privé). La Classe 4 réfère à la manière dont les formations se positionnent dans un espace géographique et institutionnel (appuyer, partenariat, contexte, position, politique, inscription, original, unique). Les rapports d'expertise maintiennent la même structure thématique que celle de la Fiche d'expertise et mobilisent un lexique institutionnalisé, c'est-à-dire des mots ou expressions figées qui préexistent dans ces documents de cadrage en tant que rubriques à renseigner.

18. Sur la notion de registre discursif, voir Adam (2004). 
Les liens entre la formation et le diplôme sont étroits. Ils tiennent au contenu même des UE et à la place donnée aux enseignements pratiques et aux interventions extérieures du monde professionnel. (Extrait d'un rapport d'expertise de l'AERES)

Ces éléments linguistiques révèlent la prédominance d'une «fonction de légitimation» (Weingart, 2004) du discours officiel que remplit l'expertise et soulignent que le moment politique ou plutôt gestionnaire l'emporte sur le moment créatif - à savoir la participation de l'expert à la définition des normes.

Que ce soit dans l'expertise universitaire ou nationale, l'usage des catégories d'évaluation tend à être réduit à un usage instrumental. L'activité menée est celle d'une normalisation : un travail de repérage et de rectification des écarts par rapport aux règles posées, dans l'objectif de produire des versions des programmes les plus « améliorées » possibles. Ce faisant, les savoirs et les discours produits par la description et l'analyse des programmes aboutissent prioritairement à des actes d'exercice de contrôle, liés aux problèmes et aux solutions recensés par le ministère et ses agences.

Cette normalisation ne va pas sans tensions. Ces tensions sont liées au «dimorphisme identitaire » qui caractérise ces experts, exerçant la fonction d'expertise parallèlement à leur fonction d'enseignant-chercheur. Le dimorphisme transparaît dans les récits de pratique des enquêtés, en particulier dans la manière dont ils s'identifient tantôt au collectif de la communauté académique ou du secteur disciplinaire, tantôt au collectif des instances politicoadministratives de l'établissement ou au collectif des experts nationaux. L'emploi des pronoms personnels «nous", "on », révèle cette position ambivalente qu'occupent ces agents, pris entre rapport professionnel à leur activité d'expertise et relations de collégialité ou d'empathie. Dans la dimension cognitive du travail d'expertise, ce dimorphisme fait surgir aussi des tensions entre, d'une part, la logique institutionnelle et, d'autre part, des logiques pratiques d'expérience ou des logiques disciplinaires. Dans l'interprétation des objectifs et des critères d'évaluation officiels, les experts mobilisent souvent des cadres sociocognitifs et de normativité qui leur sont propres. Ils s'opposent par exemple à la normalisation qu'impose l'évaluation par le taux d'insertion professionnelle des diplômés d'une formation, en inventant des indicateurs informels tenant compte de la spécificité des secteurs disciplinaires. Ils mobilisent leur expérience pratique d'enseignant-chercheur pour appréhender la place du stage professionnel dans une formation, non seulement comme expérience du marché du travail et préparant à l'emploi mais aussi comme exercice devant être articulé à l'enseignement dans une perspective d'articulation théorie-pratique. Or, ces tensions semblent s'affaiblir, voire même être passées sous silence au nom du principe de l'efficience de l'activité évaluative19. Les interprétations

19. Cette polyphonie semble se résoudre souvent par des compromis opérés par les experts euxmêmes. Un exemple, dans le cas des experts du CEVU, est celui de la séparation nette entre les 
individuelles, locales, sociales, ne sont pas réinvesties dans l'espace de production des normes pour la recontextualisation curriculaire.

\section{Vers une contribution du discours expert à la réforme de l'enseignement supérieur?}

Questionner le rôle de l'expertise dans la réforme des formations universitaires permet de donner une consistance propre au travail institutionnel de recontextualisation curriculaire. Mettre la focale analytique sur le discours de l'évaluation, comme nous l'avons fait, c'est observer la dimension pragmatique du cadrage normatif de l'action. Car ce que nous avons qualifié de « discours de l'évaluation » ne renvoie pas à une représentation spécifique du réel ni à une catégorie spécifique d'acteurs-producteurs. Il est à la fois champ de pratiques, instruments, logique d'action, catégorisations cognitives. II n'est pas un élément extérieur à l'action, mais il fait partie intégrante du dispositif qui construit et met en œuvre le changement à l'Université.

Le discours expert s'inscrit dans une relation étroite avec le discours politique officiel et, dans la fonction spécifique qu'il remplit, il contribue à matérialiser l'action sur les curricula dans les pratiques universitaires. L'Université, sous la force performative du discours politique, tend vers la rationalisation à la fois de ses objectifs et de ses activités. L'expertise des formations, sous l'égide du principe de l'efficacité, permet d'évaluer et de veiller à la mise en œuvre de ce discours. Tout cela prend forme dans la redéfinition des disciplines, et produit des effets sur leurs programmes et leurs savoirs (Stavrou, 2012). En ce sens, le travail d'évaluation se réalise actuellement comme un dispositif de contrôle qui se trouve surplombé par sa dimension institutionnelle-gestionnaire.

Comme le souligne Ogien (2007), ce modèle gestionnaire implique deux risques. Le premier renvoie au risque de la réduction de la dimension politique du débat sur l'enseignement universitaire, c'est-à-dire des différences et des conflits dans la définition des problèmes et de leurs solutions pratiques, affaiblis derrière les règles et les objets techniques et procéduraux. Le second risque désigne la prédominance de la valeur d’échange de ce travail, la recherche de l'efficacité, au détriment de ce qu'il aurait pu ou peut produire en tant que

deux rôles : le rôle de fonctionnaire au service de son employeur, l'État, qui l'emporte dans la pratique du travail expert sur le rôle de représentant élu-militant. Dans d'autres cas, les tensions se résolvent de par la pérennisation des fonctions politico-administratives de ces agents, conduisant souvent à une reconfiguration de leur identité professionnelle et de la conception qu'ils ont du travail d'universitaire : à la fois travail scientifique, pédagogique mais aussi travail administratif et politique. La réduction de la polyphonie s'explique également par des éléments organisationnels, tels que l'inscription de l'activité évaluative dans une forte hiérarchie administrative, la faiblesse ou la formalisation accrue des espaces-temps d'échange collectif en amont et en aval des expertises, ne laissant pas beaucoup de place à la visibilisation et à l'explicitation des tensions dans l'appréhension des objectifs et des moyens pour les réaliser. 
travail collectif de circulation et d'accroissement des savoirs, de réflexion et d'action sur l'enseignement universitaire. Ces deux clôtures semblent présentes dans le dispositif que nous avons examiné.

Or, les constats de notre recherche peuvent être étendus au-delà du cas de la France, et au-delà du système d'évaluation actuellement mis en place ${ }^{20}$. L'analyse permet de mettre en avant, en même temps que les clôtures, les ouvertures que pourrait présenter un travail institutionnel sur les curricula, avec la contribution d'un système d'expertise. Un tel travail pourrait en effet donner droit de cité à la question des programmes et des problèmes de la transmission des savoirs dans l'enseignement supérieur. Et il pourrait potentiellement acquérir une fonction autre que régulatrice : permettre une réflexivité collective et la construction du débat, ainsi que leur réinvestissement par l'institution.

\section{Références}

ADAM Jean-Michel, 2004, La linguistique textuelle. Des genres de discours aux textes, Paris, Nathan.

Austin John L., 1970, Quand dire c'est faire, Paris, Le Seuil.

AKRICH Madeleine, 1987, «Comment décrire les objets techniques?», Techniques et culture, n०9, p. 49-64.

BAKHTINe Mikhail M. (Valentin N. Volochinov), 1977, Le marxisme et la philosophie du langage. Essai d'application de la méthode sociologique en linguistique, Paris, Minuit.

BaLl Stephen J., 2008, The Education Debate, Bristol, Policy Press.

Bernstein Basil, 2007, Pédagogie, contrôle symbolique et identité. Théorie, recherche, critique, Québec, PUL (trad. de l'anglais par G. Ramognino-Le Déroff et P. Vitale).

ChARLIER Jean-Émile, Croché Sarah, 2003, «Le processus de Bologne, ses acteurs et leurs complices ", Éducation et sociétés, nº 12, p.13-34.

CROCHÉ Sarah, 2006, «Qui pilote le processus de Bologne?», Éducation et sociétés, vol. II, no 18 , p. 203-217.

- 2009, "Évolution d'un projet d’Europe sans Bruxelles. Le cas du processus de Bologne», Éducation et sociétés, vol. II, n² 24, p.11-27.

Cussó Roser, 2008, "Quand la Commission européenne promeut la société de la connaissance », Mots. Les langages du politique, n88, p. 39-52.

Cussó Roser, GobIN Corinne, 2008, «Du discours politique au discours expert. Le changement politique mis hors débat?», Mots. Les langages du politique, n88, p. 5-11.

DuCROT Oswald, 1984, Le dire et le dit, Paris, Minuit.

Foucault Michel, 1971, L'ordre du discours, Paris, Gallimard.

20. Dans le nouveau projet de loi, qui se prépare en 2013, l'AERES serait remplacée par un Haut Conseil de l'évaluation. Pour autant, cela ne prédit en rien un changement du rôle de l'expertise dans la réforme de l'enseignement supérieur. 
GARCIA Sandrine, 2007, «L'Europe du savoir contre l'Europe des banques? La construction de l'espace européen de l'enseignement supérieur », Actes de la recherche en sciences sociales, vol. I-II, nº166-167, p. 80-93.

Gobin Corinne, Deroubaix Jean-Claude, 2010, "L'analyse du discours des organisations internationales. Un vaste champ encore peu exploré », Mots. Les langages du politique, $n^{\circ} 94$, p. 107-114.

KRIEG-PLANQUe Alice, 2009, La notion de «formule » en analyse du discours, Besançon, Presses universitaires de Franche-Comté.

LASCOUMES Pierre et Le GALÈs Patrick éd., 2004, Gouverner par les instruments, Paris, Presses de la FNSP.

LeSSARd Claude, Bourdoncle Raymond, 2002, «Qu'est-ce qu'une formation professionnelle universitaire? Conceptions de l'université et formation professionnelle» (Note de synthèse), Revue française de pédagogie, $\mathrm{n}^{0}$ 139, p. 131-154.

MolıNo Jean, 1997, «À propos de l'argumentation en sciences humaines », Revue européenne des sciences sociales, vol.XXXV, nº 107, p.7-19.

Munck Jean (de), 1999, L'institution sociale de l'esprit, Paris, PUF.

MUSSELIN Christine, 2001, La longue marche des universités françaises, Paris, PUF.

OGIEN Albert, 2007, "La gouvernance, ou le mépris du politique », Cités, vol. IV, n³ 32 , p.137-155.

RAVINET Pauline, 2009, "Comment le processus de Bologne a-t-il commencé? La formation de la vision de l'Espace européen d'enseignement supérieur en 1998 », Éducation et sociétés, n² 24, p. 29-44.

- 2011, "La coordination européenne "à la bolognaise”. Réflexions sur l'instrumentation de l'Espace européen d'enseignement supérieur ", Revue française de science politique, vol. LXI, nº1, p. 23-49.

StAVRou Sophia, 2012, Réforme de l'Université et transformations curriculaires. Des activités de recontextualisation aux effets sur les savoirs, thèse de doctorat, AixMarseille Université.

Weingart Peter, 2004, "Expertise scientifique et responsabilité politique. Les paradoxes de la science en politique», Les sciences sociales à l'épreuve de l'action, B. Zimmermann éd., Paris, MSH, p.91-118.

\section{Textes officiels}

Commission européenne, "Soutenir la croissance et les emplois. Un projet pour la modernisation des systèmes d'enseignement supérieur en Europe», SEC (2011), 1063 final.

Conférence des ministres de l'Enseignement supérieur (communiqué), «Réaliser l'espace européen de l'enseignement supérieur», 19 septembre 2003, Berlin.

Décret du 8 avril 2002 portant application au système français d'enseignement supérieur de la construction de l'Espace européen de l'enseignement supérieur, JORF no 84 du 10 avril 2002. 
Direction générale de l'Enseignement supérieur (DGES), Circulaire du 20 juin 2005, «Campagne de contractualisation Vague $A$ ».

Loi n 68-978 du 12 novembre 1968 dite Edgar Faure d'orientation de l'enseignement supérieur.

Loi no 84-52 du 26 janvier 1984 dite Savary sur l'enseignement supérieur.

Loi n 2007-1199 du 10 août 2007 relative aux libertés et responsabilités des universités. 